\title{
The predictive value of 18F-FDG-PET early evaluation in patients with metastatic gastric adenocarcinoma treated with chemotherapy plus cetuximab
}

Francesca Di Fabio ${ }^{1}$, Carmine Pinto ${ }^{1}$, Fabiola L. Rojas Llimpe ${ }^{1}$, Stefano Fanti $^{2}$, Paolo Castellucci ${ }^{2}$, Ciro Longobardi ${ }^{1}$, Vita Mutri $^{1}$, Chiara Funaioli ${ }^{1}$, Francesca Sperandi $^{1}$, Stefania Giaquinta ${ }^{1}$, and Andrea A. Martoni ${ }^{1}$

${ }^{1}$ Medical Oncology Unit, S. Orsola-Malpighi Hospital, Via Albertoni 15-40138 Bologna, Italy

${ }^{2}$ Nuclear Medicine Unit, S. Orsola-Malpighi Hospital, Bologna, Italy

\begin{abstract}
Background. The aim of the study was to evaluate whether the therapy-induced reduction of the ${ }^{18} \mathrm{~F}$-fluorodeoxyglucose positron emission tomography (18F-FDG-PET) maximum standardized uptake value in patients with advanced gastric adenocarcinoma treated with chemotherapy plus cetuximab could predict the objective response and outcome early during the treatment.

Methods. The study was performed as a part of a phase II trial evaluating cetuximab plus the leucovorin/5-fluorouracil/ irinotecan (FOLFIRI) regimen. The objective response was evaluated according to the response evaluation criteria in solid tumors (RECIST) every 6 weeks. The early metabolic response evaluated by 18F-FDG-PET was assessed according to our own evaluated cutoff value $(<35 \%)$ after receiver operating characteristic (ROC) analysis.

Results. Twenty of 22 patients had positive baseline 18FFDG-PET. The best RECIST response was: complete response (CR), 3; partial response (PR), 9; stable disease (SD), 8. Twelve patients $(60 \%)$ were classified as metabolic responders and 8 (40\%) as nonresponders. At the median follow-up time of 11 months, median time to disease progression (TTP) and overall survival (OS) for early metabolic responders versus nonresponders were 11 versus 5 months $(P=0.0016)$ and 16 versus 6 months $(P=0.1493)$, respectively.

Conclusion. The early metabolic response evaluated by $18 F-$ FDG-PET predicted the clinical outcome in this series of patients with advanced gastric cancer treated with chemotherapy plus cetuximab.
\end{abstract}

Key words PET • Advanced gastric adenocarcinoma · Cetuximab

\section{Introduction}

Gastric cancer is the fourth most common cancer worldwide [1]. In spite of a sharp decline in gastric cancer

Offprint requests to: F. Di Fabio

Received: June 8, 2007 / Accepted: October 8, 2007 incidence during the second half of the twentieth century, it remains the second leading cause of cancer mortality in the world. Gastric cancer has a poor prognosis, as many patients $(40 \%-60 \%)$ have advanced disease at the time of diagnosis and there are many recurrences after tumor resection [2,3].

Many therapy combinations have demonstrated significant advantages in the pooled median survival time, even though patient prognosis remains very poor, with a median survival of only 7 to 10 months [4]. New studies with chemotherapy plus targeted therapies have recently been published [5,6]. These new combination therapies require an evaluation of the balance between a slight improvement in prognosis, treatment-associated toxicities, and the high cost of new drugs. It has become necessary to select the pool of patients who can draw benefits from these new treatments.

In recent years, ${ }^{18} \mathrm{~F}$-fluorodeoxyglucose positron emission tomography (18F-FDG-PET) has been investigated in order to detect primary tumors and metastatic disease, as a staging procedure, and during the followup of gastrointestinal tumors [7-9]. Several studies have also evaluated the role of 18F-FDG-PET in monitoring early response to neoadjuvant chemotherapy in different locally advanced tumors [10-13]. The predictive value of 18F-FDG-PET was observed in patients with locally advanced gastric or gastroesophageal cancer treated with neoadjuvant chemotherapy [14-16], but no studies have evaluated the role of early PET in metastatic gastric cancer.

The aim of this study was to evaluate prospectively whether early therapy-induced changes in 18F-FDGPET uptake in patients with advanced gastric cancer treated with chemotherapy plus cetuximab (Erbitux; Merck, Darmstadt, Germany) could predict the objective response and the patient outcome early in the course of treatment. 


\section{Patients and methods}

\section{Patients}

18F-FDG-PET imaging was performed as a part of a phase II trial evaluating the leucovorin/5-fluorouracil/ irinotecan (FOLFIRI) regimen in combination with cetuximab in patients with untreated advanced gastric or gastroesophageal junction adenocarcinoma (FOLCETUX Study) [6]. The study's eligibility criteria were age 18 years or more, Karnofsky performance status (KPS) 70 or more, life expectancy 3 months or more, no previous treatment with chemotherapy or radiation therapy, epithelial growth factor receptor (EGFR) expression in primary and/or metastatic tumor demonstrated by immunohistochemistry (PharmDx kit System; DakoCytomation, Glostrup, Denmark), and measurable disease. All the patients provided written informed consent for the study including consent for all the radiological and 18F-FDG-PET scan examinations. The study was approved by the local hospital's ethics committee.

\section{Treatment}

Patients received cetuximab at an initial dose of $400 \mathrm{mg} /$ $\mathrm{m}^{2} \mathrm{IV}$, followed by weekly doses of $250 \mathrm{mg} / \mathrm{m}^{2}$; plus irinotecan $180 \mathrm{mg} / \mathrm{m}^{2} \mathrm{IV}$, followed by 5 -fluorouracil $400 \mathrm{mg} / \mathrm{m}^{2}$ as a bolus and $600 \mathrm{mg} / \mathrm{m}^{2}$ IV continuous infusion for $22 \mathrm{~h}$ on days 1-2 (FOLFIRI regimen) every 2 weeks, for a maximum of 24 weeks, then cetuximab alone (maintenance therapy) was allowed in patients with a complete response (CR), partial response (PR), and stable disease (SD). Surgery for locally advanced gastric cancer could be performed during the study after at least 12 weeks of treatment.

\section{PET imaging}

18F-FDG-PET imaging was performed at baseline and was repeated after 6 weeks of treatment. As well, in some patients, 18F-FDG-PET imaging was repeated every 6 weeks during the treatment. The 18 F-FDGPET scan was carried out with a standard procedure [17]: each patient fasted for at least $6 \mathrm{~h}$, and in the absence of antidiabetic therapy, was given a $5.3 \mathrm{MBq} / \mathrm{kg}$ injection of 18 F-FDG. After the injection, patients were hydrated and the uptake phase was $60 \mathrm{~min}$. The scan was performed using a dedicated PET/computed tomography (CT) tomograph (PET/CT Discovery LS; GE Healthcare, Waukesha, WI, USA): the acquisition time was in two-dimensional (2D) mode, $4 \mathrm{~min} / \mathrm{bed}$ position, and attenuation correction was performed with a CT-based method $(120 \mathrm{kV}, 80 \mathrm{~mA})$. These CT parameters are standard for PET/CT studies. The $18 \mathrm{~F}-$ FDG-PET scan was read by two nuclear medicine phy- sicians and the report written up by consensus: any area of increased $18 \mathrm{~F}-\mathrm{FDG}$ uptake was taken into account for evaluation. The metabolic activity of each pathological lesion was measured using the maximum standardized uptake value (SUV) method. The SUV was calculated on the basis of the following formula: tissue concentration $(\mathrm{MBq} / \mathrm{g})$ / injected dose $(\mathrm{MBq})$ / body weight $(\mathrm{g})$. SUV was measured before and after treatment at any tumor site; the difference between the studies was calculated as the percentage change of baseline SUV (delta SUV).

\section{Response evaluation}

The objective response was evaluated both clinically and by $\mathrm{CT}$ scan according to the response evaluation criteria in solid tumors (RECIST) [18]. Complete response (CR) was defined as the complete disappearance of all target tumor lesions; PR as at least a $30 \%$ decrease in the sum of the longest diameter (LD) of target lesions, taking as reference the smallest sum of the LD; progressive disease (PD), as at least a $20 \%$ increase in the sum of the LD of target lesions, taking as reference the smallest sum of the LD recorded from the time the treatment started or the appearance of one or more new lesions; and SD was defined as neither sufficient shrinkage to qualify for PR nor sufficient increase to qualify for PD, taking as reference the smallest sum of the LD. The early metabolic response was evaluated by comparing the SUV after 6 weeks of therapy to the baseline SUV. We had previously performed receiver operating characteristic (ROC) analysis to determine our optimal delta SUV cutoff [19]. Metabolic responder patients were defined on the basis of the delta SUV cutoff.

\section{Statistical analysis}

All quantitative data values are expressed as medians. Time to disease progression (TTP) was defined as the time from the beginning of the study until time of disease progression or death from any cause. Patients who had no evidence of PD were censored at the date of the last follow-up. Overall survival (OS) was calculated as the time from the beginning of the study until death from any cause; patients who were alive at the date of last follow-up were censored on that date. Sensitivity, specificity, and positive and negative predictive values of a metabolic response in 18F-FDG-PET for the best response prediction were calculated using standard formulae. The optimum cutoff value for differentiation of metabolic responders and nonresponders was defined by the point on the ROC curve with the minimum distance from the $0 \%$ false-positive rate and the $100 \%$ true-positive rate. Actuarial TTP and OS curves were 
generated using the Kaplan and Meier method [20]. Statistical comparisons between different groups of patients were performed with the log-rank test. In addition, $95 \%$ confidence intervals (CI) for these parameters were calculated. All tests were two-sided and were performed at the 5\% level of significance, using SPSS for Windows, version 9.0 (SPSS, Chicago, IL, USA).

\section{Results}

From November 2004 to December 2005, 22 of 38 patients who were enrolled in the FOLCETUX study [6] had at least two consecutive 18F-FDG-PET scans, performed at baseline and after 6 weeks. A total of 111 18F-FDG-PET scans were performed (median per patient, 4; range, 2 to 8 ).

The patients' characteristics (Table 1) were as follows: 16 men, 6 women; median age, 64.5 years; median KPS, 90; primary tumor site, gastroesophageal junction, $9.1 \%$, gastric $90.9 \%$; intestinal histological subtype, $63.6 \%$; nonintestinal, $36.4 \%$ (6 of these with signet-ring cells); locally advanced disease, $13.6 \%$; metastatic disease, $86.4 \%$; and number of organs involved, one, $27.3 \%$, two, $63.6 \%$, and more than two, $9.1 \%$.

Baseline 18F-FDG-PET was negative in 2 (9.1\%) patients: they had metastatic signet-ring cell carcinoma.
Twenty patients $(90.9 \%)$ were evaluable for the present analysis.

The best objective response according to the RECIST was: 3 (15\%) CR, 9 (45\%) PR, and 8 (40\%) SD; no PD was observed. Twelve of the 20 patients were considered to be responders. After the ROC analysis (Fig. 1), a reduction of FDG uptake by more than 35\% (after 6 weeks) was found to provide the highest accuracy for the differentiation of radiological responders and nonresponders; so the early metabolic response was: 12 $(60 \%)$ responders and $8(40 \%)$ nonresponders. Table 2 shows the data for each patient, inclusive of TTP and OS at the last follow-up.

An early metabolic response correctly predicted the best RECIST response in 10 of the 12 responder patients and in 6 of the 8 nonresponder patients (Table 3). This resulted in a sensitivity and specificity of $83 \%$ (95\% CI, 62 to 104) and $75 \%$ (95\% CI, 45 to 105), respectively. Positive and negative predictive values for best RECIST response were $83 \%$ (95\% CI, 62 to 104) and 75\% (95\% CI, 45 to 105), respectively. The overall early PET accuracy to predict best RECIST response was $80 \%$ (95\% CI, 62 to 98; Table 4). No correlation was demonstrated between median SUV at baseline 18F-FDG-PET and objective response; the median SUV at baseline 18FFDG-PET was 10.9 (range, 7.6 to 29) in CR/PR patients and 9.6 (range, 6.4 to 29.8) in SD patients.

Table 1. Patient characteristics

\begin{tabular}{|c|c|c|}
\hline \multirow[b]{2}{*}{ Characteristics } & \multicolumn{2}{|c|}{$n=22$} \\
\hline & No. of patients & Percentage \\
\hline \multicolumn{3}{|l|}{ Sex } \\
\hline Male & 16 & 72.7 \\
\hline Female & 6 & 27.3 \\
\hline \multicolumn{3}{|l|}{ Age, years } \\
\hline Median & \multicolumn{2}{|c|}{64.5} \\
\hline Range & \multicolumn{2}{|c|}{$39-74$} \\
\hline \multicolumn{3}{|l|}{ Karnofsky performance status } \\
\hline Median & \multirow{2}{*}{\multicolumn{2}{|c|}{$\begin{array}{c}90 \\
70-100\end{array}$}} \\
\hline Range & & \\
\hline \multicolumn{3}{|l|}{ Primary tumor site } \\
\hline Gastroesophageal junction & 2 & 9.1 \\
\hline Stomach $^{\mathrm{a}}$ & 20 & 90.9 \\
\hline \multicolumn{3}{|l|}{ Histology } \\
\hline Intestinal adenocarcinoma & 14 & 63.6 \\
\hline Nonintestinal adenocarcinoma ${ }^{\mathrm{b}}$ & 8 & 36.4 \\
\hline \multicolumn{3}{|l|}{ Disease status } \\
\hline Locally advanced & 3 & 13.6 \\
\hline Metastatic & 19 & 86.4 \\
\hline \multicolumn{3}{|l|}{ No. of organs involved } \\
\hline 1 & 6 & 27.3 \\
\hline 2 & 14 & 63.6 \\
\hline$>2$ & 2 & 9.1 \\
\hline
\end{tabular}

${ }^{a}$ Antrum/pylorus, 9/20 (45.0\%); corpus, 11/20 (55.0\%)

${ }^{\mathrm{b}}$ Signet-ring cell carcinoma, 6/22 (27.3\%); mucinous, $1 / 22(4.5 \%)$ 
Table 2. Patient response and outcome $(n=22)$

\begin{tabular}{|c|c|c|c|c|c|c|c|}
\hline Patient no. & $\begin{array}{l}\text { Baseline } \\
\text { SUV }\end{array}$ & $\begin{array}{l}\text { SUV at } \\
6 \text { weeks }\end{array}$ & $\begin{array}{c}\text { Delta SUV } \\
(\%)\end{array}$ & $\begin{array}{l}\text { PET response } \\
\text { at } 6 \text { weeks }\end{array}$ & $\begin{array}{l}\text { Best RECIST } \\
\text { response }\end{array}$ & $\begin{array}{c}\text { TTP } \\
\text { (months) }\end{array}$ & $\begin{array}{c}\text { OS } \\
\text { (months) }\end{array}$ \\
\hline 1 & 18 & 0 & 100.00 & MR & CR & $18+$ & $18+$ \\
\hline 2 & 29 & 0 & 100.00 & MR & CR & $17+$ & $17+$ \\
\hline 3 & 10.4 & 3 & 71.15 & MR & CR & $9+$ & $9+$ \\
\hline 4 & 12.1 & 0 & 100.00 & MR & PR & 8 & 12 \\
\hline 5 & 8 & 2.6 & 67.50 & MR & PR & 3 & 9 \\
\hline 6 & 12 & 6 & 50.00 & MR & PR & 13 & 17 \\
\hline 7 & 19.2 & 11.5 & 40.10 & MR & PR & 8 & 11 \\
\hline 8 & 8.7 & 4.6 & 47.13 & MR & PR & 3 & 3 \\
\hline 9 & 10.1 & 4.2 & 58.42 & MR & PR & 11 & $11+$ \\
\hline 10 & 7.6 & 4.9 & 35.53 & MR & PR & $9+$ & $9+$ \\
\hline 11 & 8 & 8 & 0.00 & NMR & PR & 8 & 18 \\
\hline 12 & 11.4 & 10.1 & 11.40 & NMR & PR & 4 & 5 \\
\hline 13 & 6.9 & 0 & 100.00 & MR & SD & 9 & 16 \\
\hline 14 & 7 & 4.6 & 34.29 & NMR & SD & 5 & 6 \\
\hline 15 & 15.7 & 10.5 & 33.12 & NMR & SD & 6 & $12+$ \\
\hline 16 & 16.8 & 12.2 & 27.38 & NMR & SD & 8 & $8+$ \\
\hline 17 & 29.8 & 9.9 & 66.78 & MR & SD & $8+$ & $8+$ \\
\hline 18 & 10.1 & 8 & 20.79 & NMR & SD & 7 & 8 \\
\hline 19 & 9 & 7 & 22.22 & NMR & SD & 3 & 4 \\
\hline 20 & 6.4 & 6.3 & 1.56 & NMR & SD & 3 & 3 \\
\hline 21 & 0 & ND & NA & NA & SD & 8 & $10+$ \\
\hline 22 & 0 & ND & NA & NA & SD & 9 & $10+$ \\
\hline
\end{tabular}

Delta SUV was defined as the percent decrease in tumor SUVmax. Metabolic responders were defined as patients achieving delta SUV $>35 \%$

SUV, standardized uptake value; PET, positron emission tomography; TTP, time to progression; OS, overall survival; MR, metabolic response; NMR, no metabolic response; CR, complete response; PR, partial response; SD, stable disease; ND, not done; NA, not applicable; TTP+, no disease progression at last follow-up; OS+, still alive at last follow-up

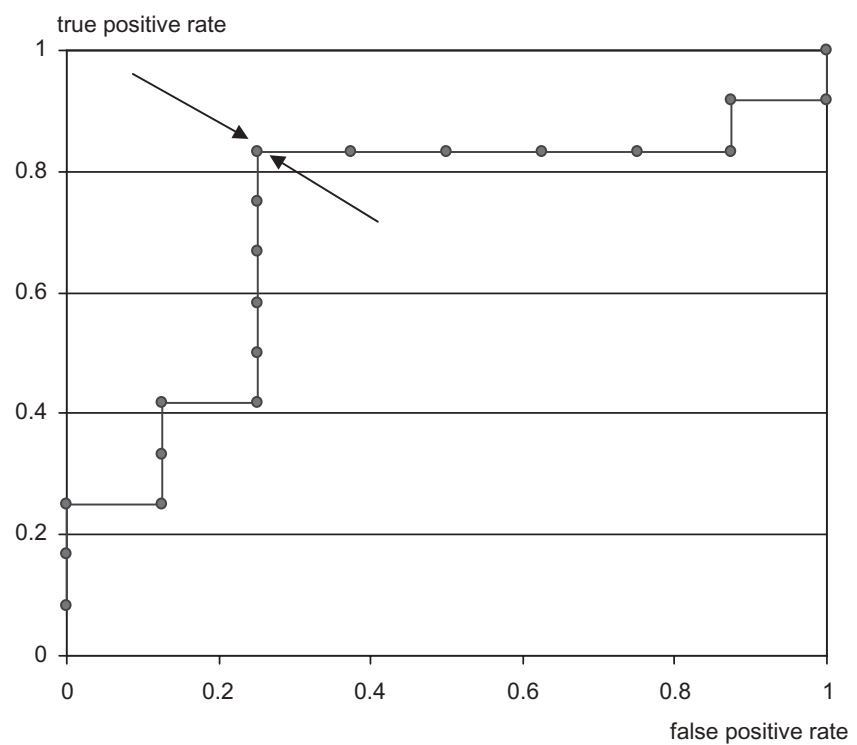

Fig. 1. Receiver operating characteristic (ROC) curve for prediction of response by ${ }^{18} \mathrm{~F}$-fluorodeoxyglucose positron emission tomography (18F-FDG-PET)

18F-FDG-PET scans were also monitored at 12 and 18 weeks in 16 and 11 patients, respectively. Figure 2 shows the median SUV variations during the treatment, according to best RECIST response. After 6 weeks,
Table 3. Early metabolic response and best RECIST response

\begin{tabular}{lccc}
\hline $\begin{array}{l}\text { RECIST } \\
\text { response }\end{array}$ & $\begin{array}{c}\text { Metabolic } \\
\text { response }^{\mathrm{a}}\end{array}$ & $\begin{array}{c}\text { No metabolic } \\
\text { response }^{\mathrm{b}}\end{array}$ & Total \\
\hline CR + PR & 10 & 2 & 12 \\
SD & 2 & 6 & 8 \\
Total & 12 & 8 & 20 \\
\hline
\end{tabular}

CR, Complete response; PR, partial response; $\mathrm{SD}$, stable disease

${ }^{a}$ Delta SUV $>35 \%$

${ }^{\mathrm{b}}$ Delta SUV $\leq 35 \%$

Table 4. Early PET accuracy to predict best RECIST response

\begin{tabular}{lcc}
\hline & No. & Percentage \\
\hline Sensitivity & $10 / 12$ & 83 \\
Positive predictive value & $10 / 12$ & 83 \\
Specificity & $6 / 8$ & 75 \\
Negative predictive value & $6 / 8$ & 75 \\
Accuracy & $16 / 20$ & 80 \\
\hline
\end{tabular}

the delta SUV was $46.8 \%$ with a trend to decrease further at 18 weeks. The curves of responders and nonresponders were already separated at 6 weeks (delta SUV 54.2 in CR/PR patients versus 30.3 in SD 


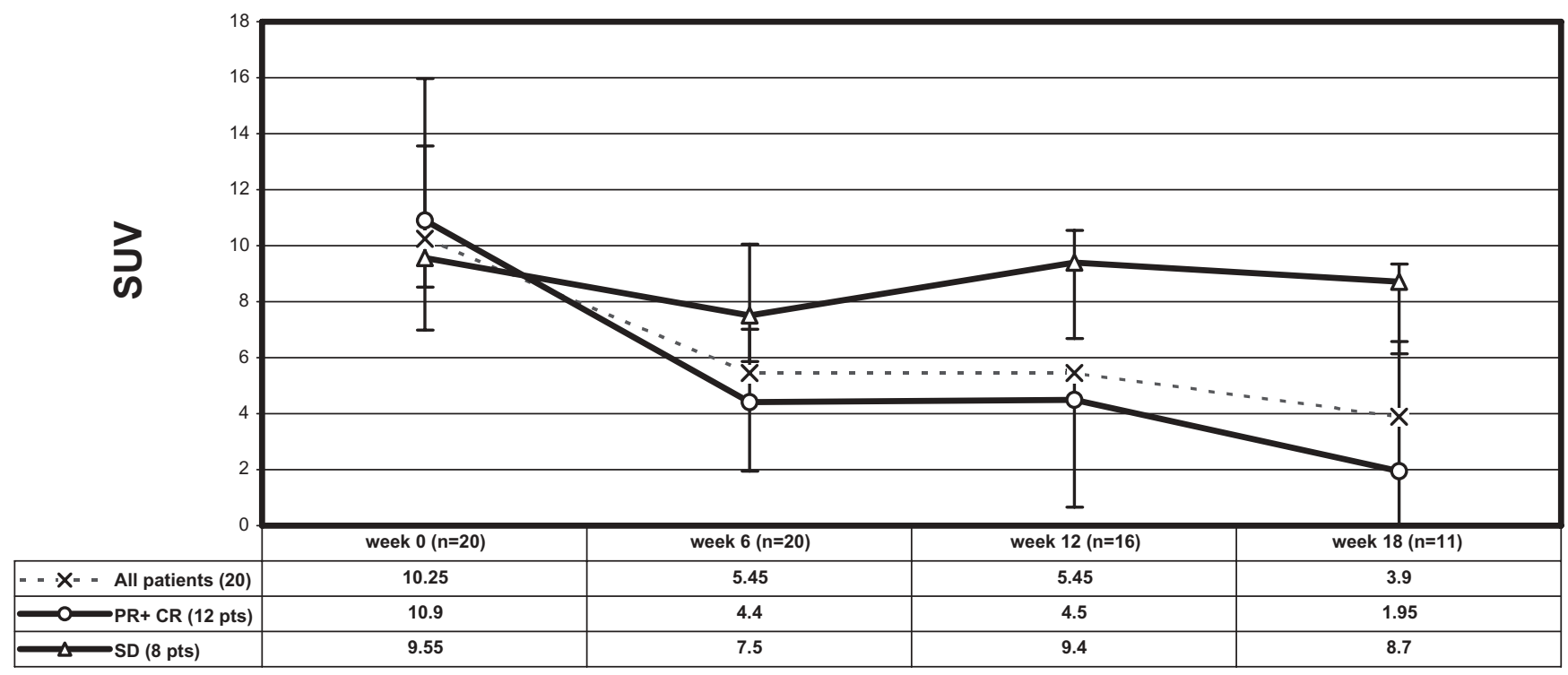

Fig. 2. Median standardized uptake value (SUVmax) variation during the treatment according to best response evaluation criteria in solid tumors (RECIST). $P R$, partial response; $C R$, complete response; $S D$, stable disease

patients), and the differences tended to increase subsequently.

The correlation between early metabolic response and the clinical outcome, as measured by TTP and OS, was analyzed. At the median follow-up time of 11 months (range, 5 to 20 months) the median TTP for metabolic responders was 11 months versus 5 months for nonresponders $(P=0.0016)$ (Fig. 3A). The median OS for early metabolic responders was 16 versus 6 months for nonresponders $(P=0.1493$; Fig. 3B).

The objective response evaluated by CT at 6 weeks (RECIST) was not significantly correlated between responders and nonresponders for TTP ( 8 months; $P=$ 0.2094 ) or OS (18 versus 16 months; $P=0.1738$ ).

\section{Discussion}

Nonresectable advanced or metastatic gastric cancer has a poor prognosis. Several combination chemotherapy regimens have been developed as first-line therapies over the past decade, with responses ranging from $20 \%$ to $40 \%$; nevertheless, median OS remains very poor, being just 7-10 months [4].

Today molecular targeting agents are the new challenge in cancer therapy, and they may have a significant impact on gastric cancer treatment [21]. In a multicenter phase II study [5], 47 untreated patients with metastatic gastric or gastroesophageal junction adenocarcinoma were treated with bevacizumab, irinotecan, and cisplatin and, at the median follow up of 12.2 months, median TTP was 8.3 months and median OS was 12.3 months.
In the Italian FOLCETUX study, 38 patients received cetuximab in combination with FOLFIRI as first-line treatment of advanced gastric cancer and, at the median follow-up time of 11 months, the median TTP was 8 months, while the median expected OS was 16 months [6]. In the FOLCETUX study, the combination of chemotherapy with a monoclonal anti-EGFR antibody was used for the first time in the treatment of advanced gastric adenocarcinoma. When the study was designed, we planned to explore the role of metabolic 18F-FDGPET evaluation to monitor the response to therapy and also to determine whether it had a possible predictive value for patient outcome.

A number of studies have shown that chemotherapy causes a marked decrease in tumor $18 \mathrm{~F}-\mathrm{FDG}$ uptake within a few weeks after the start of therapy, in different tumor types, such as lymphoma, esophagus, head and neck, lung, breast, and gastric cancer [15, 22-26]; in some of these studies the early metabolic 18F-FDG-PET response allowed for the prediction of both an objective response and patient survival [12]. Many studies have reported the predictive value of early $18 \mathrm{~F}-\mathrm{FDG}-\mathrm{PET}$ for histopathological response, prognosis, and recurrence in patients with gastric or gastroesophageal junction cancer who received preoperative chemotherapy [14-16, 27]. As Ott et al. [16] reported, in a prospective study of early 18F-FDG-PET evaluation (after 14 days) including 65 patients with locally advanced gastroesophageal junction cancer treated by neoadjuvant therapy, the metabolic responders showed a high histopathological response rate (44\%), with a 3-year survival rate of $70 \%$, as compared with a low histopathological 

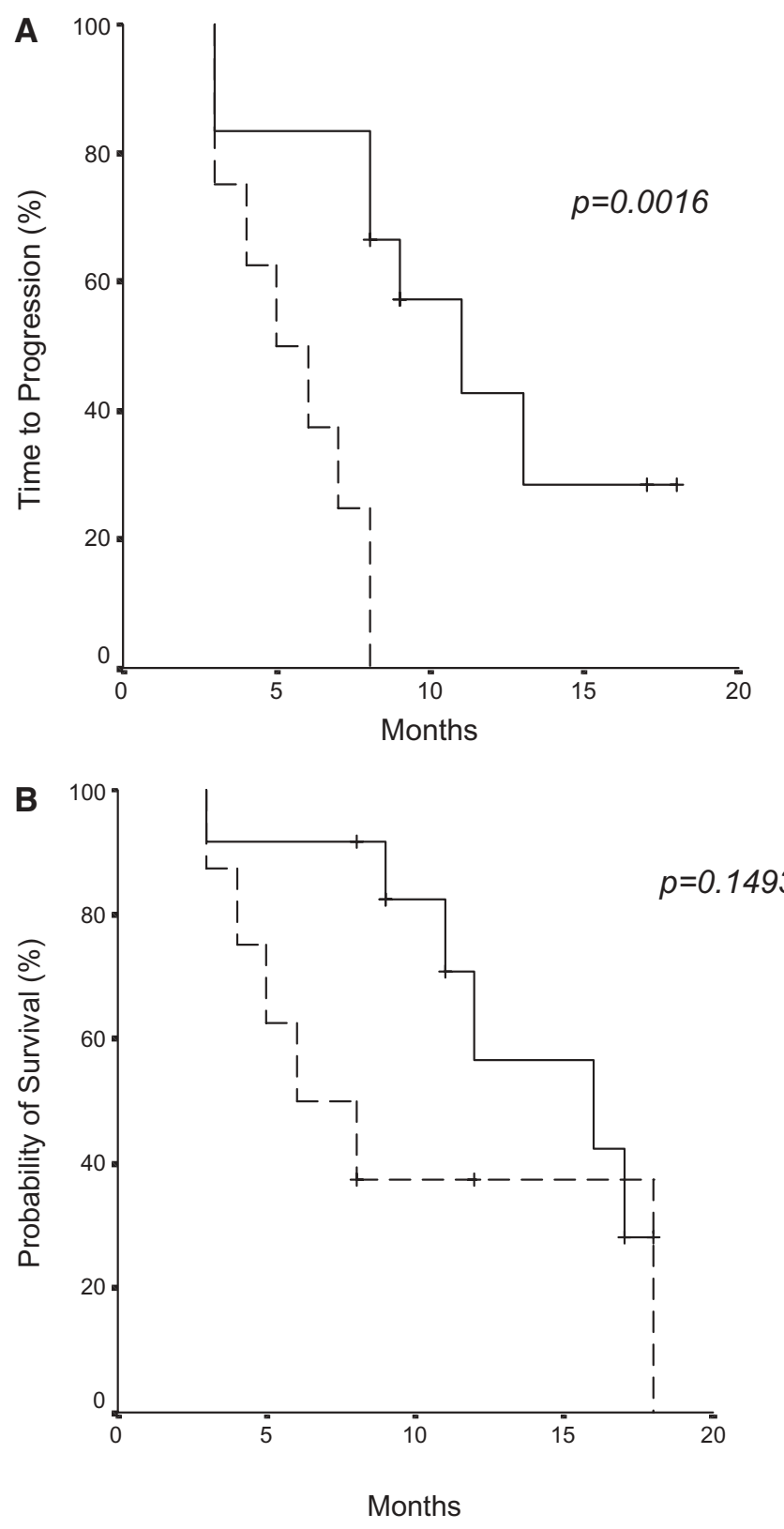

Fig. 3A,B. Kaplan-Meier plots, showing $\mathbf{A}$ time to progression and $\mathbf{B}$ overall survival in early metabolic responders $(n=$ 12 ; solid lines) and nonresponders $(n=8$; dotted lines)

response rate, of $5 \%(P=0.001)$ and a 3-year survival rate of $35 \%(P=0.01)$ in nonresponders.

Our study concerned patients with advanced gastric carcinoma and, to our knowledge, no other report on 18F-FDG-PET early response evaluation is available in this setting. The monitoring of 18F-FDG-PET every 6 weeks during the treatment enabled us to distinguish patients who would respond objectively from those who would not (Fig. 2). This separation was evident early on, at the first re-evaluation (week 6). Because the most interesting application of $18 \mathrm{~F}-\mathrm{FDG}-\mathrm{PET}$ as a predictive tool in clinical practice is its early evaluation of treatment effect, our attention and analysis focused on studying the predictive value of 18F-FDG-PET at 6 weeks. In our series, 18F-FDG-PET predicted the objective response, according to the RECIST, in 10 of 12 responders and in 6 of 8 nonresponders, with an accuracy of $80 \%$. This value would suggest a role of early 18F-FDG-PET evaluation in predicting objective response in clinical practice. As well, in our study the early 18F-FDG-PET evaluation predicted TTP and OS better than the early standard objective RECIST evaluation. As the objective response is a surrogate treatment endpoint, in this clinical setting the results shown in Fig. 3 suggest an interesting role for 18F-FDG-PET in the evaluation of new treatment regimens, including chemotherapy and biological drugs.

In the present study, the role of early 18 F-FDG-PET evaluation was that of selecting a small number of nonmetabolic responder patients with significantly worse TTP and a trend of shorter OS than metabolic responders.

Early identification of nonresponder patients could be of clinical importance in reducing the side-effects and avoiding the costs of an ineffective therapy, as well as for performing early treatment adjustments.

In conclusion, early metabolic response evaluated by 18F-FDG-PET predicted the clinical outcome in a small series of patients treated with chemotherapy plus cetuximab. These data are preliminary and our observations need to be validated in larger prospective series in order to provide the basis for clinical trials of early metabolic response-guided treatment.

Acknowledgments We thank Dr. Gaetano Compagnone (Medical Physics Unit, S. Orsola-Malpighi Hospital, Bologna, Italy) for his assistance and collaboration in the statistical analysis.

\section{References}

1. Kamangar F, Dores GM, Anderson WF. Patterns of cancer incidence, mortality, and prevalence across five continents: defining priorities to reduce cancer disparities in different geographic regions of the world. J Clin Oncol 2006;24:2137-50.

2. Hansson LE, Sparen P, Nyren O. Survival in stomach cancer is improving: results of a nationwide population-based Swedish study. Ann Surg 1999;230:162-9.

3. Kelley JR, Duggan JM. Gastric cancer epidemiology and risk factors. J Clin Epidemiol 2003;56:1-9.

4. Wagner AD, Grothe W, Haerting J, Kleber G, Grothey A, Fleig WE. Chemotherapy in advanced gastric cancer: a systematic review and meta-analysis based on aggregate data . J Clin Oncol 2006;24:2903-9.

5. Shah MA, Ramanathan RK, Ilson DH, Levnor A, D'Adamo D, O'Reilly E, et al. Multicenter phase II study of irinotecan, cisplatin, and bevacizumab in patients with metastatic gastric or gastroesophageal junction adenocarcinoma. J Clin Oncol 2006; 24:5201-6. 
6. Pinto C, Di Fabio F, Siena S, Cascinu S, Rojas Llimpe FL, Ceccarelli $\mathrm{C}$, et al. Phase II study of cetuximab in combination with FOLFIRI in patients with untreated advanced gastric or gastroesophageal junction adenocarcinoma (FOLCETUX Study). Ann Oncol 2007;18:510-7.

7. Mochiki E, Kuwano H, Katoh H, Asao T, Oriuchi N, Endo K. Evaluation of 18F-2-deoxy-2-fluoro-D-glucose positron emission tomography for gastric cancer. World J Surg 2004;28:247-53.

8. Rosenbaum SJ, Stergar H, Antoch G, Veit P, Bockisch A, Kühl H. Staging and follow-up of gastrointestinal tumors with PET/CT. Abdom Imaging 2006;31:25-35.

9. Kim SK, Kang KW, Lee JS, Kim HK, Chang HJ, Choi JY, et al. Assessment of lymph node metastases using 18F-FDG PET in patients with advanced gastric cancer. Eur J Nucl Med Mol Imaging 2006;33:148-55.

10. Kostakoglu L, Goldsmith SJ. 18F-FDG PET evaluation of the response to therapy for lymphoma and for breast, lung, and colorectal carcinoma. J Nucl Med 2003;44:224-39.

11. Kostakoglu L, Goldsmith SJ. PET in the assessment of therapy response in patients with carcinoma of the head and neck and of the esophagus. J Nucl Med 2004;45:56-68.

12. Weber WA. Use of PET for monitoring cancer therapy and for predicting outcome. J Nucl Med 2005;46:983-95.

13. Rousseau C, Devillers A, Sagan C, Ferre L, Bridji B, Campion L, et al. Monitoring of early response to neoadjuvant chemotherapy in stage II and III breast cancer by $\left[{ }^{18} \mathrm{~F}\right]$ fluorodeoxyglucose positron emission tomography. J Clin Oncol 2006;24:5366-72.

14. Weber WA, Ott K, Becker K, Dittler HJ, Helmberger H, Avril NE, et al. Prediction of response to preoperative chemotherapy in adenocarcinomas of the esophagogastric junction by metabolic imaging. J Clin Oncol 2001;19:3058-65.

15. Ott K, Fink U, Becker K, Stahl A, Dittler HJ, Busch R, et al. Prediction of response to preoperative chemotherapy in gastric carcinoma by metabolic imaging: results of a prospective trial. J Clin Oncol 2003;21:4604-10.

16. Ott K, Weber WA, Lordick F, Becker K, Busch R, Hermann K, et al. Metabolic imaging predicts response, survival, and recurrence in adenocarcinomas of the esophagogastric junction. J Clin Oncol 2006;24:4692-8.
17. Fanti S, Franchi R, Battista G, Monetti N, Canini R. PET and PETCT. State of the art and future prospects. Radiol Med 2005;11: $1-15$.

18. Therasse P, Arbuck SG, Eisenhauer EA, Wanders J, Kaplan RS, Rubistein $\mathrm{L}$, et al. New guidelines to evaluate the response to treatment in solid tumors. J Natl Cancer Inst 2000;92:205-16.

19. Metz CE. Some practical issues of experimental design and data analysis in radiologic ROC studies. Invest Radiol 1989;24: 234-45.

20. Kaplan EL, Meier P. Nonparametric estimation of incomplete observations. J Am Stat Assoc 1958;53:457-81.

21. Becker JC, Muller-Tidow C, Serve H, Domschke W, Pohle T. Role of receptor tyrosine kinases in gastric cancer: new targets for a selective therapy. World J Gastroenterol 2006;12:3297-305.

22. Schelling M, Avril N, Nährig J, Kuhn W, Römer W, Sattler D, et al. Positron emission tomography using $\left[{ }^{18} \mathrm{~F}\right]$ fluorodeoxyglucose for monitoring primary chemotherapy in breast cancer. J Clin Oncol 2000;18:1689-95.

23. Smith IC, Welch AE, Hutcheon AW, Miller ID, Payne S, Chilcott F, et al. Positron emission tomography using $\left[{ }^{18} \mathrm{~F}\right]$-fluorodeoxy-Dglucose to predict the pathologic response of breast cancer to primary chemotherapy. J Clin Oncol 2000;18:1676-88.

24. Kostakoglu L, Coleman M, Leonard JP, Kuji I, Zoe H, Goldsmith SJ. PET predicts prognosis after one cycle of chemotherapy in aggressive lymphoma and Hodgkin's disease. J Nucl Med 2002;43:1018-27.

25. Brun E, Kjellen E, Tennvall J, Ohlsson T, Sandell A, Perfekt R, et al. FDG PET studies during treatment: prediction of therapy outcome in head and neck squamous cell carcinoma. Head Neck 2002;24:127-35.

26. Weber WA, Petersen V, Schmidt B, Tyndale-Hines L, Link T, Peschel C, et al. Positron emission tomography in non-small-cell lung cancer: prediction of response to chemotherapy by quantitative assessment of glucose use. J Clin Oncol 2003;21:2651-7.

27. Ott K, Weber WA, Fink U, Helmberger H, Becker K, Stein HJ, et al. Fluorodeoxyglucose-positron emission tomography in adenocarcinomas of the distal esophagus and cardia. World J Surg 2003;27:1035-9. 